\title{
Physical activity and exercise capacity in patients with moderate COPD exacerbations
}

\author{
Ayedh D. Alahmari', Beverly S. Kowlessar ${ }^{2}$, Anant R.C. Patel ${ }^{1}$, Alex J. Mackay ${ }^{1}$, \\ James P. Allinson², Jadwiga A. Wedzicha ${ }^{2}$ and Gavin C. Donaldson ${ }^{2}$ \\ Affiliations: ${ }^{1}$ Centre for Respiratory Medicine, University College London, Royal Free Campus, London, UK. \\ ${ }^{2}$ UK Airways Disease Section, National Heart and Lung Institute, Imperial College London, London, UK.
}

Correspondence: Ayedh D. Alahmari, Centre for Respiratory Medicine, University College London, Royal Free Campus, Rowland Hill Street, Hampstead, London NW3 2PF, UK. E-mail: ayed.alahmariahotmail.com

ABSTRACT Little is known about changes in physical activity during moderate (out-patient managed) exacerbations.

6-min walking distance (6MWD) was measured during 50 exacerbations when the patients were stable, and at 3 and 7 days post-exacerbation presentation. At similar time points, quadriceps maximum voluntary contraction (QMVC) was measured during 47 different exacerbations. Physical activity (SenseWear; Bodymedia Inc., Pittsburgh, PA, USA) was recorded over 2 consecutive-week periods post-presentation.

6MWD fell from a median $422 \mathrm{~m}$ when stable to $373 \mathrm{~m}$ on day $3(\mathrm{p}=0.001)$. Similarly, QMVC fell from 32.6 versus $29.7 \mathrm{~kg}(\mathrm{p}=0.026)$. Falls in $6 \mathrm{MWD}$ were associated with a rise in $\mathrm{C}$-reactive protein $(\mathrm{r}=-0.364$; $\mathrm{p}=0.041)$ and increased Functional Assessment of Chronic Illness Therapy-Fatigue (FACIT-F) $(r=-0.44$; $\mathrm{p}=0.013$ ). Light physical activity was $2.18 \mathrm{~h} \cdot$ day $^{-1}$ during the first week post-exacerbation and was less over week 2 ( $1.98 \mathrm{~h} \cdot \mathrm{day}^{-1}$; $\left.\mathrm{p}=0.009\right)$. Patients who had attended pulmonary rehabilitation had smaller changes in 6MWD than those who had not attended $(-35.0$ versus $-114.9 \mathrm{~m} ; \mathrm{p}=0.013)$. Falls in physical activity were correlated with higher depression scores $(\mathrm{rho}=-0.51 ; \mathrm{p}=0.006)$.

These findings indicate that exercise capacity and muscle strength fall at exacerbation in chronic obstructive pulmonary disease patients who are treated at home and are free to maintain normal activity.

@ERSpublications

Falls in physical capacity and muscle strength during COPD exacerbations managed in the community http://ow.ly/ZtU45

This article has supplementary material available from erj.ersjournals.com

Received: July 092015 | Accepted after revision: March 032016 | First published online: April 282016

Support statement: The study was part funded by the Medical Research Council (UK) Patient Cohort grant G0800570/1. A.D. Alahmari is funded by the Saudi Ministry of Higher Education, the Kingdom of Saudi Arabia. The funding bodies had no input on any aspect of this study. Funding information for this article has been deposited with FundRef.

Conflict of interest: Disclosures can be found alongside the online version of this article at erj.ersjournals.com

Copyright OERS 2016 


\section{Introduction}

Chronic obstructive pulmonary disease (COPD) patients can experience episodes of acute worsening of respiratory symptoms termed exacerbations. During these episodes, breathlessness increases, the perception of fatigue worsens $[1,2]$, markers of systemic inflammation rise [3-5], and patients spend less time outside their home [6] and take fewer steps during the day [7]. In severe cases, exacerbation results in hospital admission when profound falls in physical activity have been described [8] together with a loss of quadriceps strength [9].

Skeletal muscle weakness has been shown to be an important predictor of exercise limitation in COPD [10]. Various contributors to muscle weakness at exacerbation have been hypothesised, including immobility, treatment with corticosteroids, nutritional deficit, oxidative stress and inflammation [11]. There have been studies of exercise capacity, physical activity and muscle strength during the very early phases of hospitalised exacerbations $[8,9]$, but few studies have examined moderate exacerbations treated in the community [12-14].

We therefore prospectively investigated whether acute changes in physical activity during naturally acquired, outpatient-treated exacerbations were associated with changes in exercise capacity, quadriceps strength, systemic inflammatory markers and fatigue levels. Some of the findings of this study have been previously reported in abstract form $[13,14]$.

\section{Methods}

\section{London COPD cohort}

Patients were recruited from the London COPD cohort, which started in 1995 to prospectively study the aetiology and pathophysiology of COPD exacerbations. Patients completed daily diary cards and were seen in clinic every 3 months when stable, and annually underwent a comprehensive medical review. Patients were also seen at exacerbation (detected using diary card entries and defined below), and 3 and 7 days later. Inclusion in the cohort required a forced expiratory volume in $1 \mathrm{~s}$ (FEV1)/forced vital capacity (FVC) $<0.7$ and a $\mathrm{FEV}_{1} \%$ predicted $<80 \%$. Patients with other primary respiratory diseases or who were unable or unwilling to complete daily diary cards were excluded. For this sub-study, which started in April 2011, we also excluded patients who required walking support (a cane or frame), who were confined to a wheel chair or who needed to carry ambulatory oxygen cylinders.

Ethics approval for this sub-study was granted from the London-Hampstead Ethics Committee (REC ref 09/H0720/8 and 11/LO/1630). Patients gave informed, written consent.

\section{Stable, clinic visits}

The patient characteristics reported in table 1 were collected when the patient was stable (non-exacerbating) and nearest in time to the start of the exacerbation, either at an annual review or a recruitment visit.

TABLE 1 Characteristics of patients participating in protocol 1 and protocol 2

Protocol 1

Protocol 2: QMVC

SenseWear armband 6MWD

\begin{tabular}{lccc}
\hline Subjects $\mathbf{n}$ & 50 & 44 & 47 \\
Age years & $72.9 \pm 8.2$ & $73.3 \pm 8.3$ & $72.4 \pm 7.8$ \\
FEV 1 \% pred & $50.7 \pm 15.1$ & $50.9 \pm 15.8$ & $50.1 \pm 17.2$ \\
FEV1 L & $1.36 \pm 0.6$ & $1.33 \pm 0.6$ & $1.24 \pm 0.5$ \\
FVC L & $2.78 \pm 0.9$ & $2.71 \pm 0.9$ & $2.81 \pm 1.0$ \\
FEV 1 /FVC \% & $48.8 \pm 13.2$ & $49.2 \pm 13.6$ & $45.2 \pm 13.6$ \\
BMI kg.m ${ }^{-2}$ & $26.6 \pm 5.6$ & $26.6 \pm 5.6$ & $25.9 \pm 5.6$ \\
6MWD m & $414 \pm 111$ & $414 \pm 111$ & $426 \pm 104$ \\
Exacerbations per year & $2(1.0-4.0)$ & $2(1.0-4.0)$ & $3(2.0-4.0)$ \\
Males & 72 & 70 & 60 \\
Smoking at recruitment & 30 & 27 & 32 \\
GOLD mild to moderate & 45 & 43 & 55 \\
GOLD severe to very severe & 55 & 57 & 42 \\
Attended PR & 60 & 64 & 62
\end{tabular}

Data are presented as mean $\pm \mathrm{SD}$, median (interquartile range) or $\%$, unless otherwise stated. SenseWear is manufactured by Bodymedia Inc., Pittsburgh, PA, USA. 6MWD: 6-min walking distance; QMVC: quadriceps maximum voluntary contraction; FEV1: forced expiratory volume in $1 \mathrm{~s} ; \%$ pred: \% predicted; FVC: forced vital capacity; BMI: body mass index; GOLD: Global Initiative for Chronic Obstructive Lung Disease; PR: pulmonary rehabilitation. 
Patients were considered to be stable 30 days after the onset of an exacerbation and $\geqslant 14$ days after ending treatment (oral corticosteroids and/or antibiotics).

When stable, FEV1 and FVC were measured with a FlowScreen II spirometer (Viasys Ltd, Tonbridge, UK) and age, height and weight were recorded. Patients were categorised as mild, moderate, severe or very severe according to the Global Initiative for Chronic Obstructive Lung Disease (GOLD) classification [12].

\section{Clinic assessments and home monitoring}

Patients were asked to perform a 6-min walking distance (6MWD) test according to American Thoracic Society (ATS) guidelines [15] with pre- and post-test measurements of heart rate and oxygen saturation; dyspnoea and fatigue levels were assessed using a Borg Scale. The quadriceps maximum voluntary contraction (QMVC) was measured in the dominant leg [16]. Patients completed the Functional Assessment of Chronic Illness Therapy-Fatigue (FACIT-F) [17] and the Hospital Anxiety and Depression Scale (HADS) questionnaires [18]. The highest possible FACIT-F score of 52 indicated no fatigue and a score of 0 indicated maximum fatigue. A maximum HADS score of 21 indicated abnormally high anxiety and depression levels. Venous blood was drawn for measurement of C-reactive protein (CRP) using a Modular Analytics E 170 Module (Roche, Burgess Hill, UK). Patients were asked if they had ever undertaken a pulmonary rehabilitation programme.

Patients who participated in the London COPD cohort were asked to use diary cards on a daily basis, recording any increase in or new respiratory symptoms and whether they had taken any oral prednisolone or antibiotics for COPD exacerbations.

\section{Exacerbations}

An exacerbation was defined as an increase in respiratory symptoms for 2 consecutive days, with at least one major symptom (dyspnoea, sputum purulence or sputum volume) plus one other major or a minor symptom (wheeze, cold, sore throat or cough). Patients were asked to call a 24 -h manned phone line to arrange an appointment as soon as their symptoms deteriorated, and generally presented in clinic within 2 days of the onset of symptoms (the day of presentation was termed day 0 ). They were then asked to re-attend clinic on days 3 and 7 post-presentation when blood was sampled, questionnaires were administered and physical tests were performed.

Exacerbation duration (recovery time) was defined as the number of days from exacerbation onset that increased respiratory symptoms were still being recorded. The first of 2 consecutive symptom-free days marked when the exacerbation had recovered [19].

\section{Protocol 1}

50 patients who presented with an exacerbation were asked to wear a SenseWear Armband 7.0 (Bodymedia Inc., Pittsburgh, PA, USA) over their left tricep continuously (except whilst washing or sleeping) for 14 days post-presentation. The SenseWear device indirectly measures energy expenditure and has previously been validated for use in COPD patients $[20,21]$. Data collected on days with incomplete recordings were excluded. Patients completed HADS and FACIT-F questionnaires. After presentation (day 0 ), patients returned to the clinic and undertook a 6MWD test on days 3 and 7. Due to changes in 6MWD over time [22], only data from patients with an exacerbation and a stable measurement in the same 12-month period were analysed. Figure S1 presents a consort diagram providing protocol details.

\section{Protocol 2}

47 patients (19 of whom had performed protocol 1) underwent QMVC testing [16] when stable and on days 3 and 7 after exacerbation presentation. These 47 exacerbations were not the same exacerbations as those in protocol 1. The equipment used to measure QMVC included a chair with a seatbelt, a strain gauge, an ankle strap and a horizontal bar. Exacerbation and stable data were collected in the same year. Figure S2 presents a consort diagram providing protocol details.

\section{Exacerbation frequency}

Patients were divided into frequent exacerbators (more than two exacerbations in the 12 months preceding the exacerbation under investigation) and infrequent exacerbators (two or fewer exacerbations) [23]. If earlier diary card data was unavailable due to recent recruitment $(n=3)$, the number of exacerbations was based on patient recall of events in the preceding year [24].

\section{Statistical analysis}

All data were analysed using SPSS (IPM SPSS statistical 22). Normally distributed data are reported as mean $\pm \mathrm{SD}$ or mean $\pm \mathrm{SEM}$, and skewed data are reported as median (interquartile range (IQR)). Data 
recorded at baseline and during recovery were compared using a paired t-test or the Wilcoxon rank-sum test. The relationship between changes in 6MWD, QMVC, FACIT-F and CRP between baseline and exacerbation were examined using Pearson correlation (r) or Spearman rank correlation (rho). Significance was taken as a p-value of $<0.05$.

\section{Results}

Table 1 shows the characteristics of the 50 COPD patients who wore the SenseWear armband and the subset of 44 patients who agreed to undergo a 6MWD test during exacerbation recovery (protocol 1). Table 1 also describes the 47 patients whose QMVC was measured at exacerbation (protocol 2).

\section{Exacerbation characteristics}

Of the 50 exacerbations in protocol 1, 35 received antibiotics and oral corticosteroids, six received antibiotics only, eight received oral corticosteroids only, and for one the patient was instructed to increase their dose of inhaled corticosteroids. All oral corticosteroid courses were 7 days and typically $30 \mathrm{mg}$ per day. The antibiotics courses were: amoxicillin (23 exacerbations), co-amoxiclav (11 exacerbations), clarithromycin (five exacerbations) and doxycycline (two exacerbations). Symptomatic recovery could be assessed from the diary cards for 46 of the 50 exacerbations; recovery took a median of 11 (range 8-16) days. $89.1 \%$ of the exacerbations involved increased dyspnoea, $60.9 \%$ increased sputum purulence and $69.6 \%$ increased sputum volume.

Of the 47 exacerbations examined in protocol 2, 33 received antibiotics and oral corticosteroids, 12 received antibiotics only, and two received oral corticosteroids only; one patient was prescribed amoxicillin and oral corticosteroids but it was left to the patient's discretion whether to take these and it is not known whether they did. Courses of oral corticosteroids were all 7 days. The antibiotics courses were: amoxicillin (23 exacerbations), co-amoxiclav (nine exacerbations), clarithromycin (seven exacerbations), doxycycline (four exacerbations), ciprofloxacin (one exacerbation) and unknown (one exacerbation). Exacerbation recovery could be assessed in 40 exacerbations and was a median of 13.5 (range 9-21.5) days; of these, 80.0\% involved increased dyspnoea, $62.5 \%$ involved increased sputum purulence and $77.5 \%$ involved increased sputum volume.

\section{Changes in exercise capacity and muscle strength with exacerbation}

Figure 1a shows that when stable, the median (IQR) 6MWD was $422 \mathrm{~m}(337-500 \mathrm{~m}$ ) but fell by $49 \mathrm{~m}$ (13.1\%) to $373 \mathrm{~m}(265-450 \mathrm{~m})$ by day $3(\mathrm{p}=0.001$, paired; $\mathrm{n}=35)$. The mean \pm SEM QMVC (figure $1 \mathrm{~b}$ ) was $32.6 \pm 2.7 \mathrm{~kg}$ when stable, but fell by $8.9 \%$ with exacerbation to $29.7 \pm 2.5 \mathrm{~kg}$ on day 3 ( $\mathrm{p}=0.026$, paired; $\mathrm{n}=29$ ). Table 2 shows that dyspnoea levels were higher on day 3 compared to when stable, both before $(\mathrm{p}=0.005)$ and after $(\mathrm{p}=0.014)$ the $6 \mathrm{MWD}$ test. Fatigue levels were also higher before the $6 \mathrm{MWD}$ on day 3 compared to the stable measurements $(\mathrm{p}=0.0051)$ but not between the stable and day 3 fatigue levels collected after the 6MWD test $(\mathrm{p}=0.098)$. There were no differences in heart rate or oxygen saturation.

Improvements in 6MWD were seen as the patient recovered between days 3 and 7, rising in paired data from median (IQR) $373 \mathrm{~m}(265-450 \mathrm{~m})$ to $415 \mathrm{~m}(290-490 \mathrm{~m})(\mathrm{p}<0.001 ; \mathrm{n}=33)$. However, QMVC was still less than the stable measurements on day $7:$ mean \pm SEM $29.1 \pm 2.8 \mathrm{~kg}$ compared with $32.2 \pm 2.7 \mathrm{~kg}$ ( $\mathrm{p}=0.019$, paired; $\mathrm{n}=29$ ).

\section{Changes in physical activity during exacerbation}

Figure 1c shows that the mean duration of energy expenditure $>2.5$ METs was greater during week 1 post-exacerbation presentation (mean \pm SEM $2.18 \pm 0.23 \mathrm{~h} \cdot \mathrm{day}^{-1}$ ) than during week $2\left(1.98 \pm 0.22 \mathrm{~h} \cdot \mathrm{day}^{-1}\right.$ ) $(\mathrm{p}=0.009, \mathrm{n}=46)$. Time spent lying down either sleeping or resting was not significantly different in week 1 $\left(11.06 \pm 0.24 \mathrm{~h} \cdot \mathrm{day}^{-1}\right)$ compared to week $2\left(11.35 \pm 0.26 \mathrm{~h} \cdot \mathrm{day}^{-1}\right)(\mathrm{p}=0.158, \mathrm{n}=46)$ (figure 1d). Four patients did not complete week 2 .

FACIT-F levels were worse at exacerbation presentation (day 0) compared with the stable state (mean \pm SEM $31 \pm 1.7$ versus $36 \pm 1.5 ; \mathrm{p}<0.001 ; \mathrm{n}=39$ ), and on day 3 ( $35 \pm 1.5$ versus $37 \pm 1.4 ; \mathrm{p}=0.037 ; \mathrm{n}=36$ ). Depression levels also were higher on day 0 (median (IQR) $5(2-7)$ versus $4(2-6)$ at the stable state; $\mathrm{p}=0.024 ; \mathrm{n}=31$ ) but had returned to stable state levels by day $3(4(2-7)$ versus $4(2-6)$ paired stable data; $\mathrm{p}=0.316 ; \mathrm{n}=28)$.

\section{Inflammatory changes during exacerbation}

Table 2 also shows that systemic inflammation rose at exacerbation with CRP increasing from median (IQR) $3(1-8) \mathrm{mg} \cdot \mathrm{L}^{-1}$ at baseline to $8(3-37) \mathrm{mg} \cdot \mathrm{L}^{-1}$ at exacerbation presentation (day 0$)(\mathrm{p}<0.001 ; \mathrm{n}=42)$. It remained elevated relative to the stable state on day $3\left(5(2-13.5) \mathrm{mg} \cdot \mathrm{L}^{-1}\right.$ versus $3(1-8.5) \mathrm{mg} \cdot \mathrm{L}^{-1} ; \mathrm{p}=0.027$; $\mathrm{n}=34)$ but by day 7 had fallen significantly from peak levels on day $0\left(8(4-42) \mathrm{mg} \cdot \mathrm{L}^{-1}\right.$ to $3(1-7) \mathrm{mg} \cdot \mathrm{L}^{-1}$; $\mathrm{p}<0.001 ; \mathrm{n}=43$ ). 

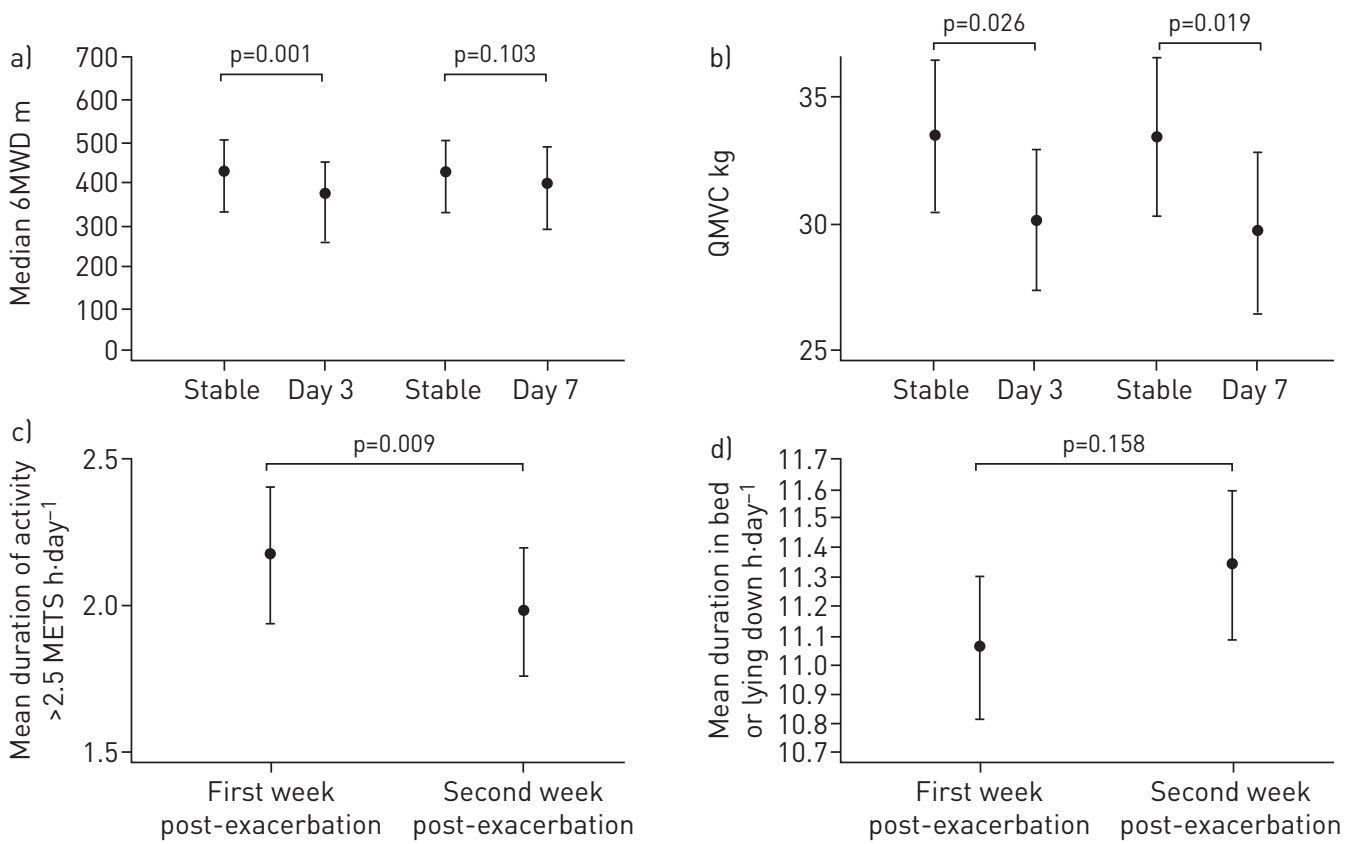

FIGURE 1 a) 6-min walking distance (6MWD) in stable patients and during exacerbation recovery. b) Quadriceps maximum voluntary contraction (QMVC) in stable patients and during exacerbation recovery. c) Duration of light activity in weeks 1 and 2 post-exacerbation. d) Duration of time in bed at night or lying down during the day in weeks 1 and 2 post-exacerbation. Dots represent the mean and whiskers represent the interquartile ranges for $6 \mathrm{MWD}$, and the standard error of the mean for quadriceps strength, exercise duration, and the time in bed or lying down. All p-values are paired.

Frequent versus infrequent exacerbators

We found that infrequent exacerbators ( $\leqslant 2$ per year) had a smaller reduction in the duration of light activity (week 1-week 2) compared with patients with frequent exacerbations (mean \pm SEM $-0.10 \pm 0.09 \mathrm{~h} \cdot$ day $^{-1}$ versus $-0.40 \pm 0.11 \mathrm{~h} \cdot \mathrm{day}^{-1}$, respectively; $\mathrm{p}=0.048 ; \mathrm{n}=46$ ) (figure $2 \mathrm{a}$ ). There were no differences between frequent and infrequent exacerbators in the change in 6MWD or QMVC (baseline to day 3) (figure $2 \mathrm{~b}$ and c).

Disease severity

There were no differences in light energy expenditure or quadriceps strength between mild-to-moderate GOLD stages and severe-to-very severe GOLD stages. However, falls in 6MWD between baseline and day 0 were significantly greater in patients with severe-to-very severe disease compared with mild-to-moderate GOLD stage patients (mean \pm SEM $-81.2 \pm 21.9 \mathrm{~m}$ versus $-24.1 \pm 13.8 \mathrm{~m}$, respectively; $\mathrm{p}=0.034$ ) (figure $2 \mathrm{e}$ ).

TABLE 2 Measurements for the 50 patients examined in protocol 1

\begin{tabular}{|c|c|c|c|c|}
\hline & Baseline & Day 0 & Day 3 & Day 7 \\
\hline 6MWD m & 422 (337-500) & & $374(265-450)$ & $415(290-490)$ \\
\hline FEV 1 L & $1.35 \pm 0.61$ & $1.26 \pm 0.57$ & $1.28 \pm 0.51$ & $1.30 \pm 0.61$ \\
\hline Pre-sulfur dioxide $\%$ & $94(92-96)$ & & $93.5(92-96)$ & $94(92-96)$ \\
\hline Post -sulfur dioxide \% & 93.5 (89-95) & & $91.0(86-96)$ & $92(88-94)$ \\
\hline Pre-dyspnoea & $0.91 \pm 0.96$ & & $1.50 \pm 1.13^{\#}$ & $1.46 \pm 1.26$ \\
\hline Post-dyspnoea & $2.63 \pm 1.26$ & & $3.21 \pm 1.04^{\pi}$ & $3.14 \pm 1.60$ \\
\hline Pre-fatigue & $0.66 \pm 1.0$ & & $1.31 \pm 1.43$ & $1.26 \pm 1.56$ \\
\hline Post-fatigue & $1.61 \pm 1.8$ & & $2.05 \pm 1.81$ & $2.26 \pm 1.92$ \\
\hline Pre-heart rate bpm & $80 \pm 12$ & & $83 \pm 13$ & $80 \pm 12$ \\
\hline Post-heart rate bpm & $94 \pm 15$ & & $97 \pm 15$ & $93 \pm 17$ \\
\hline CRP $\mathrm{mg} \cdot \mathrm{dL}^{-1}$ & $3(1-8)$ & $8(3-37)$ & $5(2-12)$ & $3(1-7)$ \\
\hline
\end{tabular}

Data are presented as median (25th and 75th percentiles) or mean \pm SD. 6MWD: 6-min walking distance; FEV1: forced expiratory volume in $1 \mathrm{~s}$; bpm: beats per minute; CRP: C-reactive protein. ${ }^{\#}: \mathrm{p}=0.0045$, day 0 versus day $3 ;{ }^{\text {१ : }} \mathrm{p}=0.0139$, day 0 versus day 3 . 
a)
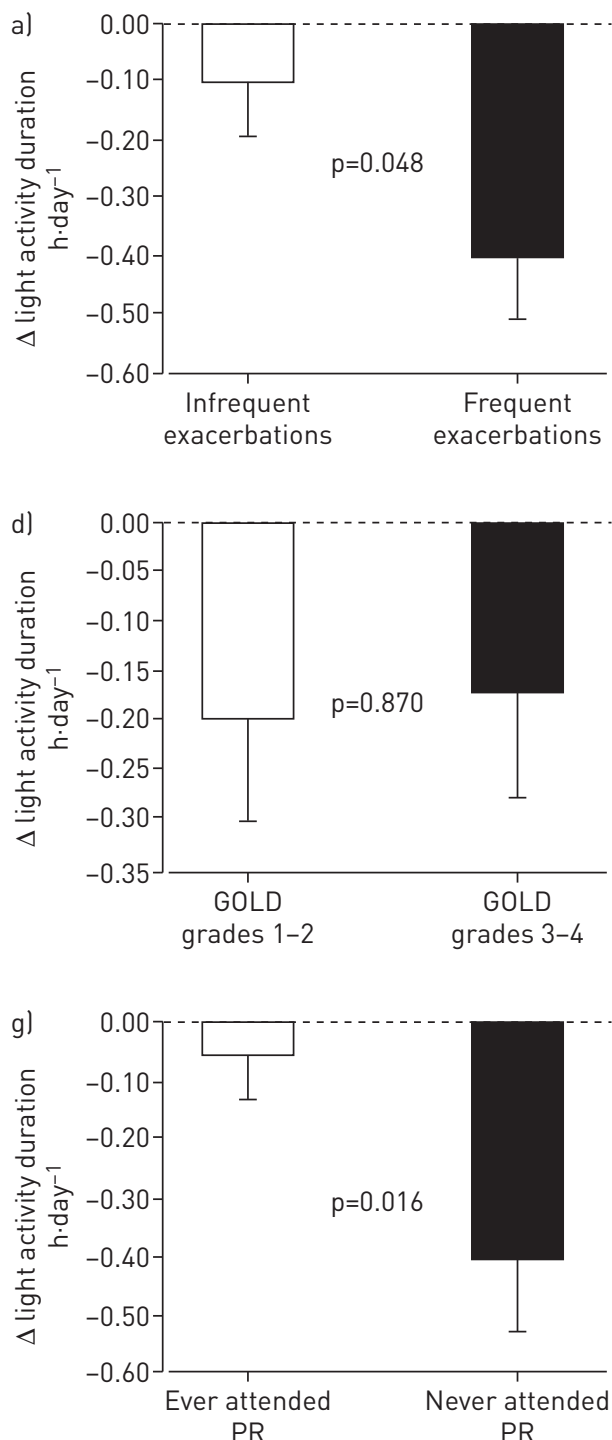
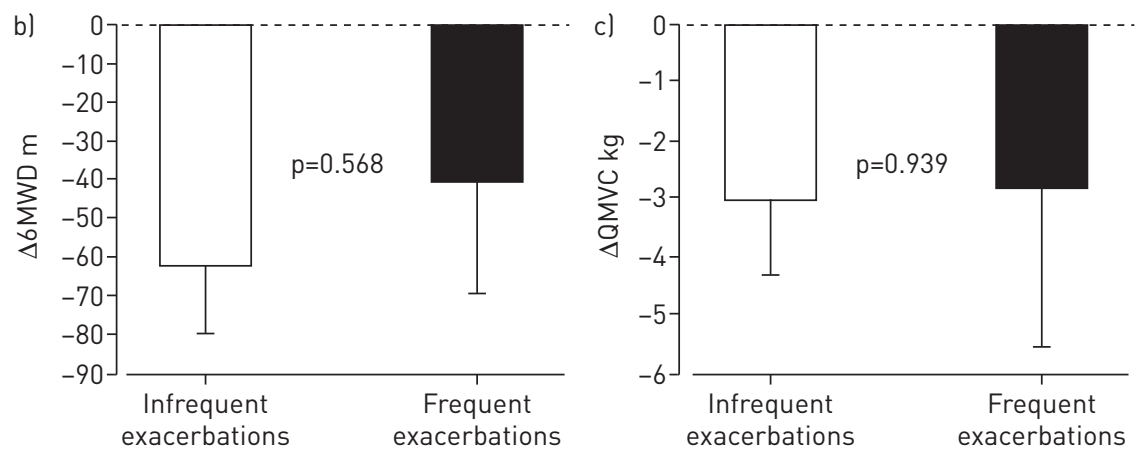
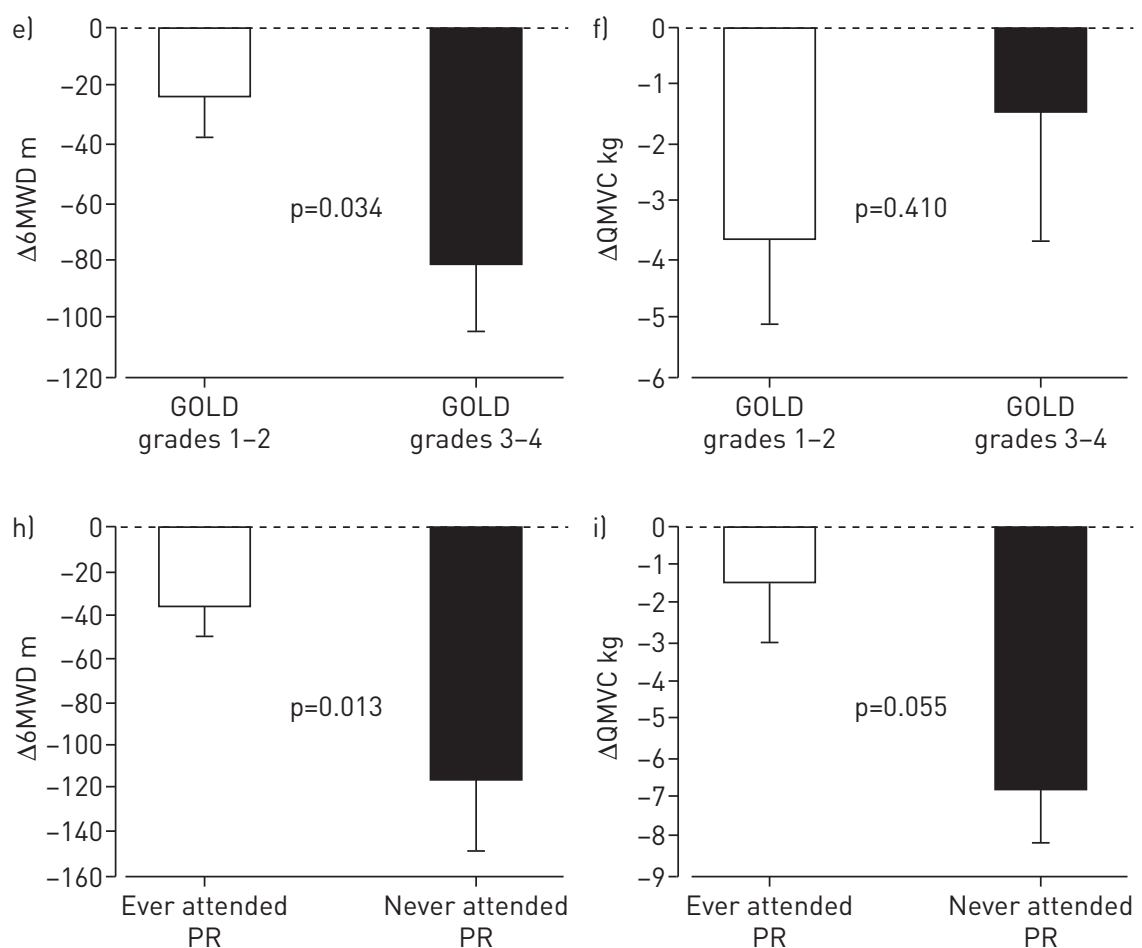

FIGURE 2 Change in light activity duration (week 1 to week 2), 6-min walking distance ( $66 \mathrm{MWD}$ ) and quadriceps maximum voluntary contraction $(\triangle \mathrm{QMVC})$ at exacerbation (day 3 to stable state) sub-divided by $\mathrm{a}-\mathrm{c}$ ) a previous history of frequent versus infrequent exacerbations, $\mathrm{d}-\mathrm{f}$ ) chronic obstructive pulmonary disease Global Initiative for Chronic Obstructive Lung Disease (GOLD) grade severity, and g-i) whether the patient had ever previously attended a pulmonary rehabilitation (PR) course.

\section{Pulmonary rehabilitation attendance}

30 of the 50 patients in protocol 1 had attended a pulmonary rehabilitation course a mean of 1.9 years prior to their exacerbation. Patients who had previously attended pulmonary rehabilitation had a smaller difference between weeks 1 and 2 in their mean \pm SEM daily duration of light activity of only $-0.06 \pm 0.07 \mathrm{~h} \cdot$ day $^{-1}$ compared with patients who had never undertaken pulmonary rehabilitation whose activity was $-0.41 \pm 0.129 \mathrm{~h} \cdot \mathrm{day}^{-1}$ lower in week 1 relative to week $2(\mathrm{p}=0.016)$ (figure $2 \mathrm{~g}$ ). Similarly, there was a smaller decrease in 6MWD with exacerbations in patients who had attended pulmonary rehabilitation compared with those who had not undertaken pulmonary rehabilitation $(-35.0 \pm 14.1 \mathrm{~m}$ and $-114.9 \pm 32.2 \mathrm{~m}$, respectively; $\mathrm{p}=0.013$ ) (figure $2 \mathrm{~h}$ ). There was also a trend to a smaller fall in quadriceps muscle strength compared with patients who had or had not attended pulmonary rehabilitation $(-1.5 \pm 1.5 \mathrm{~kg}$ and $-6.8 \pm 1.4 \mathrm{~kg}$, respectively; $\mathrm{p}=0.055)$ (figure $2 \mathrm{c}$ ).

\section{Relationship between changes in fatigue and depression with physical activity}

Increasing levels of fatigue (a fall in FACIT-F score) were associated with a greater reduction in 6MWD between baseline and day $3(\mathrm{r}=0.435 ; \mathrm{p}=0.013)$ (figure $3 \mathrm{a}$ ), and higher depression scores were associated with larger falls in light energy expenditure between the first and second week (figure $3 \mathrm{~b}$; rho $=-0.507$; $\mathrm{p}=0.006 ; \mathrm{n}=28$ ). There was no relationship between changes in the duration of light energy expenditure and changes in fatigue $(\mathrm{r}=0.15 ; \mathrm{p}=0.441)$. 

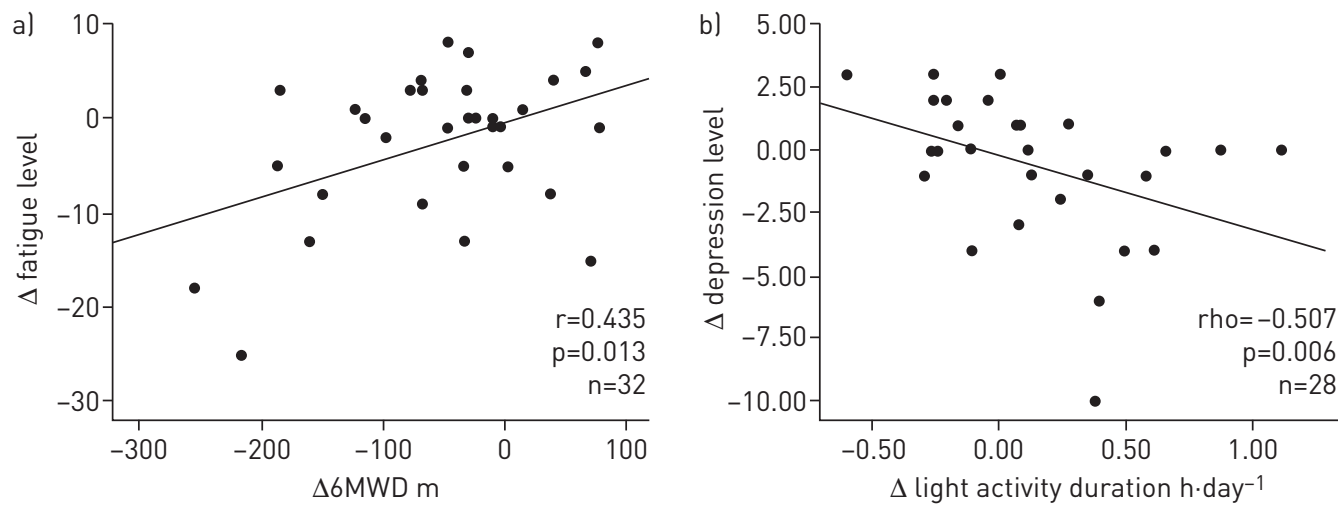

FIGURE 3 a) Changes with exacerbation in both fatigue and 6-min walking distance ( $\triangle 6 \mathrm{MWD}$ ) between baseline and day 3 post-exacerbation. b) Changes in depression scores and light activity duration between week 2 and week 1.

\section{Changes in systemic inflammation and exercise capacity at exacerbation}

Figure 4 shows that larger falls in 6MWD between stable and day 3 measurements were seen in patients with the greatest rises in CRP $(r=-0.364 ; \mathrm{p}=0.041 ; \mathrm{n}=32)$ (figure $4 \mathrm{a})$. When measured at exacerbation presentation, patients with higher CRP levels had larger falls in 6MWD on day 3 compared with the stable state $(\mathrm{r}=-0.390 ; \mathrm{p}=0.023 ; \mathrm{n}=34$ ) (figure $4 \mathrm{~b}$ ).

Patients at exacerbation presentation with a rise in CRP greater than the group median of $2.55 \mathrm{mg} \cdot \mathrm{dL}^{-1} \mathrm{had}$ a significantly lower mean \pm SEM duration of light activity 1 week post-exacerbation $\left(1.63 \pm 0.26 \mathrm{~h} \cdot \mathrm{day}^{-1}\right)$ than patients with a below median rise in CRP whose light activity was $\left(2.69 \pm 0.37 \mathrm{~h} \cdot \mathrm{day}^{-1} ; \mathrm{p}=0.026\right)$ (figure 5).

\section{Discussion}

This study showed that 6MWD and QMVC were reduced by $13.1 \%$ and $8.9 \%$, respectively, during community-treated COPD exacerbations. These declines in physical performance indicators were accompanied by changes in the perception of fatigue and increases in systemic inflammation (CRP) levels. We also found that changes in the duration of "light" energy expenditure during exacerbation recovery were related to increases in depression levels.

Skeletal muscle dysfunction and wasting are amongst the most important extra-pulmonary manifestations of COPD. In healthy individuals, muscle fatigue limits exercise capacity. In COPD patients, we observed increased levels of fatigue but also breathlessness, which may have caused patients to stop exercising before fatigue was noticeable [25]. We observed that the decrements in exercise capacity at exacerbation were larger in severe-to-very severe GOLD grade COPD patients than in mild-to-moderate COPD patients. The importance of breathlessness is supported by studies that show physical activity related to lung hyperinflation [26], since hyperinflation is an important determinant of breathlessness. The 5-unit increase in FACIT-F scores with exacerbation is consistent, but smaller, than the 8.3-unit increase we have previously reported [1]. It could be that patients volunteering to undergo exercise testing at exacerbation do so more willingly because they are not experiencing large increases in fatigue.
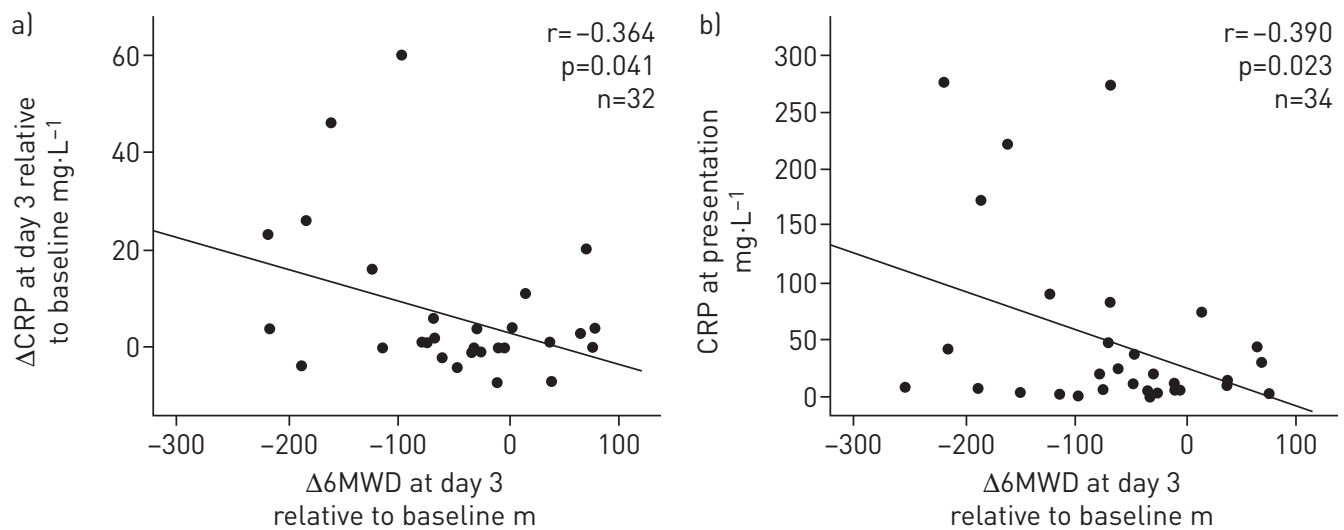

FIGURE 4 Changes in 6-min walking distance (6MWD) at exacerbation (from day 3 to stable state) in relation to a) changes in C-reactive protein (CRP) (from day 3 to day 0 ) and b) CRP at exacerbation presentation. 


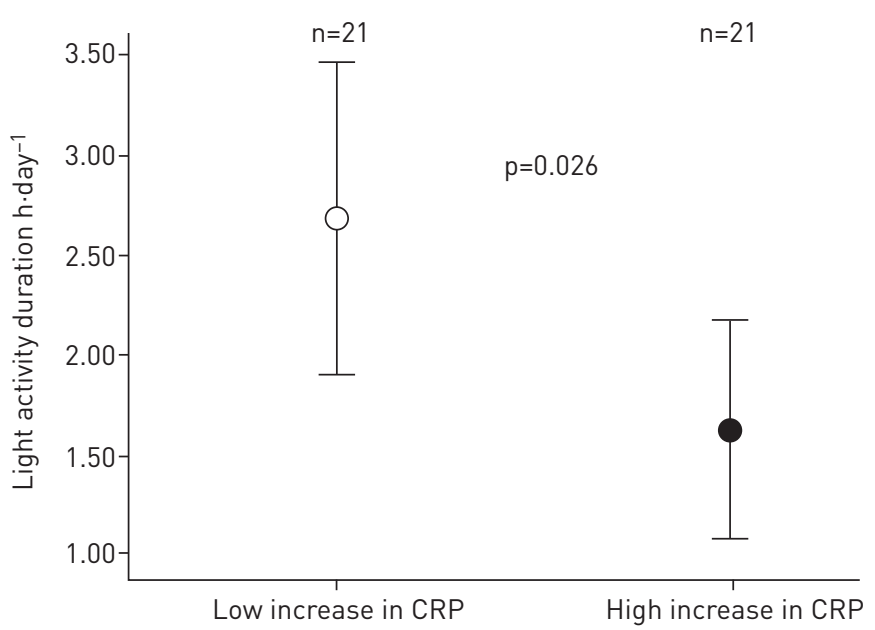

FIGURE 5 Difference in light activity per day during week 1, between patients with low and high increases in C-reactive protein (CRP) from baseline to exacerbation presentation. CRP median: $2.55 \mathrm{mg} \cdot \mathrm{dL}^{-1}$.

Currently, there is interest in a possible link between muscle weakness and systemic inflammation, as the latter could be modified by anti-inflammatory therapies. The evidence so far is limited to cross-sectional studies that may be confounded by disease severity and exacerbation frequency [27-29]. Evidence from patients hospitalised for an acute exacerbation maybe difficult to interpret as bed rest in healthy people is known to decrease exercise capacity [30,31]. Our study examined moderate exacerbations during which patients were at liberty to engage in their normal daily activities. This category of exacerbation comprises the majority of exacerbation events that involve healthcare resources. We found a significant association between the rise in CRP at exacerbation and the decrease in 6MWD between baseline and 3 days post-presentation. It is unlikely that CRP has a direct effect on muscle but its rise at exacerbation will be correlated with increases in other acute-phase proteins, such as interleukin (IL)-1 $\beta$, tumour necrosis factor (TNF)- $\alpha$ and $\alpha_{1}$-antichymotrypsin (ACP). Chronic treatment with TNF- $\alpha$ or IL- 1 in rats decreases muscle protein content [32] possibly through interference with myoblast differentiation [33] and high levels of ACP are associated with loss of hand grip strength [34]. Sputum levels of TNF- $\alpha$ and serum levels of ACP increase at exacerbation $[35,36]$. This association should be treated cautiously and other experimental designs are required to test the hypothesis but our findings suggest that further work is merited.

In this study, frequent exacerbations were a patient phenotype associated with a greater reduction at exacerbation in the duration of light energy expenditure. We have previously reported that patients with frequent exacerbations experience a significantly faster decline in both the amount of time they spend outside the home [6] and their daily step-count [7]. Thus, this group of patients appears at greatest risk of exercise impairment. Patients with frequent exacerbations have higher modified Medical Research Council dyspnoea scores [37] and may therefore be more likely to experience levels of exacerbation-related breathlessness that are sufficient to limit activity. We also observed that increases in depression scores between weeks 2 and 1 correlated with reduced energy expenditure in week 2 compared with week 1 . Higher depression scores are seen in patients who spend less time outdoors [38]. It is possible that patients confined indoors become progressively more depressed, which would explain why they were less inclined to engage in light or heavier exercise in week 2 compared with week 1.

In those patients who had attended a pulmonary rehabilitation course at some point, reductions at exacerbation in the duration of "light" energy expenditure and 6MWD were significantly smaller than those who had not attended a course. The effect on QMVC was not significant $(p=0.055)$ but the direction and consistency with all three different outcome measures was noteworthy. During pulmonary rehabilitation, patients are educated about their disease and undergo exercise training to improve muscle strength and desensitise them to dyspnoea [39, 40]. We believe this is the first time an effect of pulmonary rehabilitation on changes in physical activity at exacerbation has been reported and it is worthy of prospective investigation, as this finding may encourage patients to undertake these programmes.

A limitation of the study is that we did not include COPD patients who used ambulatory oxygen or walking supports, and our results should not be extrapolated to these patient groups. It was sometimes difficult to persuade patients with an exacerbation to undertake exertional activity and thus data were missing at various time points. EATON et al. [41] also noticed this reluctance to engage in exercise at exacerbation, with only $97(42 \%)$ of 228 eligible patients agreeing to participate in a randomised, controlled trial of early pulmonary rehabilitation at exacerbation. We therefore cannot rule out a bias 
caused by patients refusing to participate if they were more disabled by the exacerbation, though this may have led us to underestimate the true effect. Another restriction is that the limited number of SenseWear devices meant it was not feasible with to prospectively collect stable data over 7 days and to ask the patient to make as a special journey to return the device. Our study could have been improved by electromagnetic stimulation of the quadriceps as this would have eliminated the perception of fatigue as a cause of muscle weakness [42].

In summary, exacerbations managed outside of hospital are associated with a decline in exercise capacity and increased muscle weakness. These changes were greater in those with more severe disease or a history of frequent exacerbations. It is possible that prior pulmonary rehabilitation may protect against loss of exercise capacity and physical activity at exacerbation, and greater rises in systemic inflammation were associated (though possibly coincidently) with a larger reduction in exercise capacity.

\section{Acknowledgements}

The authors would like to thank the patients in the London COPD cohort who contributed to the study. The authors are grateful to COPDMAP (www.copdmap.com) and Professor M. Polkey (Royal Brompton Hospital, London, UK) for sharing the quadriceps maximum voluntary contraction methodology and for his insightful comments on the manuscript.

\section{References}

1 Baghai-Ravary R, Quint JK, Goldring JJ, et al. Determinants and impact of fatigue in patients with chronic obstructive pulmonary disease. Respir Med 2009; 103: 216-223.

2 Seemungal TA, Donaldson GC, Bhowmik A, et al. Time course and recovery of exacerbations in patients with chronic obstructive pulmonary disease. Am J Respir Crit Care Med 2000; 161: 1608-1613.

3 Hurst JR, Donaldson GC, Perera WR, et al. Use of plasma biomarkers at exacerbation of chronic obstructive pulmonary disease. Am J Respir Crit Care Med 2006; 174: 867-874.

4 Wedzicha JA, Seemungal TA, MacCallum PK, et al. Acute exacerbations of chronic obstructive pulmonary disease are accompanied by elevations of plasma fibrinogen and serum IL-6 levels. Thromb Haemost 2000; 84: 210-215.

5 Bhowmik A, Seemungal TA, Sapsford RJ, et al. Relation of sputum inflammatory markers to symptoms and lung function changes in COPD exacerbations. Thorax 2000; 55: 114-120.

6 Donaldson GC, Wilkinson TM, Hurst JR, et al. Exacerbations and time spent outdoors in chronic obstructive pulmonary disease. Am J Respir Crit Care Med 2005; 171: 446-452.

7 Alahmari AD, Patel AR, Kowlessar BS, et al. Daily activity during stability and exacerbation of chronic obstructive pulmonary disease. BMC Pulm Med 2014; 14: 98.

8 Pitta F, Troosters T, Probst VS, et al. Physical activity and hospitalization for exacerbation of COPD. Chest 2006; 129: 536-544.

9 Spruit MA, Gosselink R, Troosters T, et al. Muscle force during an acute exacerbation in hospitalised patients with COPD and its relationship with CXCL8 and IGF-I. Thorax 2003; 58: 752-756.

10 Cotes JE, Zejda J, King B. Lung function impairment as a guide to exercise limitation in work-related lung disorders. Am Rev Respir Dis 1988; 137: 1089-1093.

11 Hopkinson NS, Man WD, Dayer MJ, et al. Acute effect of oral steroids on muscle function in chronic obstructive pulmonary disease. Eur Respir J 2004; 24: 137-142.

12 Vestbo J, Hurd SS, Agusti AG, et al. Global strategy for the diagnosis, management, and prevention of chronic obstructive pulmonary disease: GOLD executive summary. Am J Respir Crit Care Med 2013; 187: 347-365.

13 Alahmari AKB, Patel A, Mackay A, et al. The relationship between exercise capacity and inflammatory markers at COPD exacerbation. Thorax 2014; 69: Suppl. 2, A19.

14 Patel AKB, Mackay A, Singh R, et al. COPD exacerbation frequency phenotype and exercise capacity. Am J Respir Crit Care Med 2013; 187: A1440.

15 ATS Committee on Proficiency Standards for Clinical Pulmonary Function Laboratories. ATS statement: guidelines for the six-minute walk test. Am J Respir Crit Care Med 2002; 166: 111-117.

16 Edwards RH, Young A, Hosking GP, et al. Human skeletal muscle function: description of tests and normal values. Clin Sci Mol Med 1977; 52: 283-290.

17 Tennant KF. Assessment of fatigue in older adults: the FACIT Fatigue Scale (version 4). Ann Longterm Care 2011; 19.

18 Zigmond AS, Snaith RP. The hospital anxiety and depression scale. Acta Psychiatr Scand 1983; 67: 361-370.

19 Donaldson GC, Law M, Kowlessar B, et al. Impact of prolonged exacerbation recovery in chronic obstructive pulmonary disease. Am J Respir Crit Care Med 2015; 192: 943-950.

20 Waschki B, Spruit MA, Watz H, et al. Physical activity monitoring in COPD: compliance and associations with clinical characteristics in a multicenter study. Respir Med 2012; 106: 522-530.

21 Patel SA, Benzo RP, Slivka WA, et al. Activity monitoring and energy expenditure in COPD patients: a validation study. COPD 2007; 4: 107-112.

22 Polkey MI, Spruit MA, Edwards LD, et al. Six-minute-walk test in chronic obstructive pulmonary disease: minimal clinically important difference for death or hospitalization. Am J Respir Crit Care Med 2013; 187: 382-386.

23 Donaldson GC, Seemungal TAR, Bhowmik A, et al. Relationship between exacerbation frequency and lung function decline in chronic obstructive pulmonary disease. Thorax 2002; 57: 847-852.

24 Quint JK, Donaldson GC, Hurst JR, et al. Predictive accuracy of patient-reported exacerbation frequency in COPD. Eur Respir J 2011; 37: 501-507.

25 Newell SZ, McKenzie DK, Gandevia SC. Inspiratory and skeletal muscle strength and endurance and diaphragmatic activation in patients with chronic airflow limitation. Thorax 1989; 44: 903-912.

26 Garcia-Rio F, Lores V, Mediano O, et al. Daily physical activity in patients with chronic obstructive pulmonary disease is mainly associated with dynamic hyperinflation. Am J Respir Crit Care Med 2009; 180: 506-512.

27 Yende S, Waterer GW, Tolley EA, et al. Inflammatory markers are associated with ventilatory limitation and muscle dysfunction in obstructive lung disease in well functioning elderly subjects. Thorax 2006; 61: 10-16. 
Broekhuizen R, Wouters EF, Creutzberg EC, et al. Raised CRP levels mark metabolic and functional impairment in advanced COPD. Thorax 2006; 61: 17-22.

29 Pinto-Plata VM, Mullerova H, Toso JF, et al. C-reactive protein in patients with COPD, control smokers and non-smokers. Thorax 2006; 61: 23-28.

30 Hung J, Goldwater D, Convertino VA, et al. Mechanisms for decreased exercise capacity after bed rest in normal middle-aged men. Am J Cardiol 1983; 51: 344-348.

31 Corcoran PJ. Use it or lose it - the hazards of bed rest and inactivity. West J Med 1991; 154: 536-538.

32 Argilés JM, Busquets S, Felipe A, et al. Molecular mechanisms involved in muscle wasting in cancer and ageing: cachexia versus sarcopenia. Int J Biochem Cell Biol 2005; 37: 1084-1104.

33 Langen RC, Schols AM, Kelders MC, et al. Inflammatory cytokines inhibit myogenic differentiation through activation of nuclear factor- $\kappa$ B. FASEB J 2001; 15: 1169-1180.

34 Schaap LA, Pluijm SMF, Deeg DJ, et al. Inflammatory markers and loss of muscle mass (sarcopenia) and strength. Am I Med 2006; 119: 526.e9-e17.

35 Aaron SD, Angel JB, Lunau M, et al. Granulocyte inflammatory markers and airway infection during acute exacerbation of chronic obstructive pulmonary disease. Am J Respir Crit Care Med 2001; 163: 349-355.

36 Crooks SW, Bayley DL, Hill SL, et al. Bronchial inflammation in acute bacterial exacerbations of chronic bronchitis: the role of leukotriene B4. Eur Respir J 2000; 15: 274-280.

37 Donaldson GC, Mullerova H, Locantore N, et al. Factors associated with change in exacerbation frequency in COPD. Respir Res 2013; 14: 79.

38 Quint JK, Baghai-Ravary R, Donaldson GC, et al. Relationship between depression and exacerbations in COPD. Eur Respir J 2008; 32: 53-60.

39 Cooper CB. Desensitization to dyspnea in COPD with specificity for exercise training mode. Int J Chron Obstruct Pulmon Dis 2009; 4: 33-43.

40 Puhan MA, Scharplatz M, Troosters T, et al. Respiratory rehabilitation after acute exacerbation of COPD may reduce risk for readmission and mortality - a systematic review. Respir Res 2005; 6: 54.

41 Eaton T, Young P, Fergusson W, et al. Does early pulmonary rehabilitation reduce acute health-care utilization in COPD patients admitted with an exacerbation? A randomized controlled study. Respirology 2009; 14: $230-238$.

42 Rutherford OM, Jones DA, Newham DJ. Clinical and experimental application of the percutaneous twitch superimposition technique for the study of human muscle activation. J Neurol Neurosurg Psychiatry 1986; 49: 1288-1291. 